\title{
Diagnósticos de Neoplasia maligna de testículos em umhospital universitário: uma análise epidemiológica no período de 5 anos.
}

Diagnosis of malignant testicle neoplasia in a university hospital: an epidemiological analysis in the 5 year perod.

\section{Carlos Egydio Ferri do Carmo ${ }^{1}$}

http://www.seer.ufms.br/in dex.php/pecibes/index

${ }^{1}$ Graduação em Urologia pela Universidade Federal de Mato Grosso do Sul no Hospital Universitário Maria Aparecida Pedrossian.

\section{Resumo}

\section{*Autor}

correspondente:

Carlos Egydio Ferri

do Carmo.

E-mail do autor: carlosegydiofc@hotmail .com

Palavras-chave:

Câncer de Testículo. Neoplasia Maligna. Orquiectomia Radical.

Key-words:

Testicular Cancer. Malignant neoplasm. Radical Orchiectomy.
O câncer de testículo, embora ser uma malignidade rara, acomete mais frequentemente homens de 15 a 35 anos. Tem sua incidência elevando-se gradativamente nos últimos anos. Sua suspeita se dá por uma massa testicular sólida, sendo a ultrassonografia escrotal o estudo de imagem inicial elegido. A orquiectomia radical é tanto diagnóstica quanto terapêutica. Dentre os tumores testiculares, os de células germinativas são o mais comum na população adulta. O seu tratamento é basicamente cirúrgico, sendo indicado, em alguns casos, a realização de ciclos quimioterápicos. Avanços substanciais no tratamento foram feitos nas últimas décadas que tornaram esta neoplasia a malignidade sólida com maior índice de cura. Com a terapêutica eficaz, a taxa de sobrevida global em cinco anos é de $98 \%$. O objetivo deste estudo é analisar, estatisticamente, o perfil epidemiológico dos pacientes submetidos a orquiectomia radical no serviço de urologia do hospital maria aparecida pedrossian no período de janeiro de 2014 a dezembro de 2019.

\section{Abstract}

Testicular cancer, despite being a rare malignancy, most often affects men aged 15 to 35 years. Its incidence has gradually increased in recent years. Its suspicion is due to a solid testicular mass, with scrotal ultrasound being the chosen initial imaging study. Radical orchiectomy is both diagnostic and therapeutic. Among testicular tumors, germ cell tumors are the most common in the adult population. Its treatment is basically surgical, being indicated, in some cases, the performance of chemotherapy cycles. Substantial advances in treatment have been made in recent decades that have made this neoplasm the solid malignancy with the highest cure rate. With effective therapy, the overall fiveyear survival rate is $98 \%$. The objective of this study is to statistically analyze the epidemiological profile of patients undergoing radical orchiectomy in the urology service of the Maria aparecida pedrossian hospital from January 2014 to December 20 


\section{Introdução}

Embora se tratar de um tipo de malignidade rara, a raridade da neoplasia no Brasil e demais localidades com prevalência global de $2 \%$, o câncer de testículo é o mundiais, onde perfaz apenas $1 \%$ a $2 \%$ dos tumores do homem, tumor sólido mais comum em homens de 15 a 35 anos e sua representando $0,1 \%$ da mortalidade câncer-específico neste incidência vem aumentando continuamente nas últimas décadas (Cappuccio et al., 2018; Smith et al., 2013). Habitualmente surge como uma massa testicular indolor unilateral no escroto (Bosl et al., 2005). Cerca de 95\% são tumores de células germinativas, categorizados como seminoma e não seminoma, principalmente pelas diferenças na história natural (Horner et al., 2009). A orquiectomia radical é tanto diagnóstica quanto terapêutica (Thompson; Blute, 2004). Avanços substanciais no tratamento feitos nas últimas décadas tornaram esta neoplasia a malignidade sólida com maior índice de cura, proporcionando um excelente prognóstico (Baird et al., 2018). Com a terapêutica eficaz, a taxa de sobrevida global em cinco anos é de $98 \%$ (National Comprehensive Cancer Center, 2018).

\section{Material e Métodos}

Trata-se de um estudo descritivo, retrospectivo, quantitativo envolvendo 24 pacientes submetidos à orquiectomia radical, pareados por faixa etária, lateralidade, distribuição por ano realizado e tipos histológicos, no período entre janeiro de 2014 e dezembro de 2019. A Pesquisa foi realizada mediante parecer consubstanciado do CEP da Universidade Federal do Mato Grosso do Sul, CAAE 28449420.1.0000.0021, número do parecer: 4.060.381.

\section{Resultados}

No período analisado, foram registrados 24 pacientes submetidos à orquiectomia radical por tumor testicular, sendo 2018 o ano de maior prevalência, com 10 casos (42\%), e 2014 e 2017, os de menor prevalência, com 2 casos cada. Constatou-se que houve predomínio da $2^{\mathrm{a}}$ e $3^{\mathrm{a}}$ décadas de vida, com pacientes entre 20-29 anos 9 (37,5\%) casos e entre 30-39 anos 7 (29\%) casos. No que tange a lateralidade, houve destaque para o lado direito, com 15 casos. Em relação aos tipos histológicos presentes no anatomopatológico foram encontrados quatro, distribuídos como: tumor de células germinativas seminomatoso em 10 casos $(42 \%)$; tumor de células germinativas mistas em 9 $(37,5 \%)$; tumor de células germinativas não seminomatoso $3(12,5 \%)$ e tumor de estroma gonadal tipo tumor de células de sertoli em 2 (8\%). Não houve nenhum óbito.

\section{Discussão}

Foram realizados 24 orquiectomias radicais no período de janeiro de 2014 a dezembro de 2019, não sendo excluído nenhum paciente, todos se enquadraram nos critérios de inclusão proposto na metodologia do presente estudo. Nas bases de dados utilizadas (pubmed, medline e up to date) para o estudo, o que prevalece na literatura são trabalhos robustos avaliando o câncer de testículo descritivamente, relatos de casos solitários e sua incidência/prevalência isoladamente em regiões mais prevalentes. mesmo sexo (Silva et al., 2017; Srougi et al., 1999).

Em relação à faixa etária dos pacientes em análise, nosso estudo mostrou um pico de incidência no período que compreende da $2^{a}$ a $3^{a}$ décadas de vida, $66,5 \%$ dos casos, corroborando com a literatura que relata mesma epidemiologia de predomínio de idade em pacientes com carcinoma testicular (Presti et al., 2014).

Dos tumores testiculares primários, $2,5 \%$ dos casos são bilaterais $(0,6 \%$ sincrônicos e $1,9 \%$ metacrônicos), e cerca de $50 \%$ deles ocorrem em homens com uma história de criptorquidia unilateral ou bilateral (Weijl et al., 2000; Maciel et al., 2012). Tanto no serviço de urologia do HumapUFMS/Ebserh, quanto nos estudos analisados há um predomínio mais comum para o lado direito do que o esquerdo, o que acompanha a incidência aumentada de criptorquidia à direita, de acordo com exposto por Presti et al., em 2014. Existem vários fatores de risco ambientais independentemente associados à neoplasia testicular, sendo a criptorquidia o principal (Cook et al., 2010).

Quando definimos a distribuição de orquiectomia radical realizada por cada ano analisado da amostra observamos um predomínio de um maior número de cirurgias no ano de 2018, 10 pacientes (42\%), mas com discreta elevação anual dos casos desde o início da nossa pesquisa. A literatura, tal qual nosso levantamento são coincidentes com esse aumento progressivo. Contudo, apesar do aumento da incidência de indivíduos com câncer de testículo, a mortalidade desses pacientes tem diminuído com o advento das melhorias de estadiamento, classificação de riscos e propostas terapêuticas (Tavora et al., 2013; Vlayen et al., 2012).

Analisando as linhagens histológicas encontradas, o tumor de células germinativas seminomatoso tipo clássico foi o mais frequente, representado por 10 casos estudados, mas quando distribuídos por seminoma e não seminoma, há um destaque para os não seminomas com 12 casos (50\%) contra 10 casos seminomas $(42 \%)$ e apenas 2 casos de tumor de estromas gonadal (8\%). Na maioria dos estudos avaliados verificou-se uma prevalência pelo tumor testicular de células germinativas (95\%) comparadas ao tumor testicular de células estromais (5\%) (Tavora et al., 2013). Dentro do tipo células germinativas há um discreto predomínio do subtipo nãoseminoma confrontado ao subtipo seminoma (45\%) (Stephenson et al., 2019). E incluso ao subtipo seminomatoso há uma maioria do tumor testicular seminomatoso clássico, com $80 \%$ dos casos descritos (Faria; Freitas, 2010). Já no subtipo não seminomatoso há destaque para a histologia mista (Dall'oglio et al., 2010). Dados que são coincidentes com os encontrados no serviço de urologia do HumapUFMS/Ebserh.

\section{Conclusão}

Com base no exposto, conclui-se que houve preferência da neoplasia pela $2^{a}$ e $3^{a}$ décadas de vida, com predomínio do tumor de células germinativas $\mathrm{O}$ volume de orquiectomia realizadas, 24 procedimentos seminomatoso e com excelente evolução clínica dos cirúrgicos na totalidade, condiz com a literatura, demostrando pacientes. Apesar da raridade, esta morbidade não pode ser 
menosprezada, visto que apresenta diagnóstico simples, tratamento bem-conceituado e excelente prognóstico.

\section{Agradecimentos}

Agradeço à Universidade Federal de Mato Grosso do Sul e ao Serviço de Residência Médica em Urologia do Hospital Universitário Maria Aparecida Pedrossian pela oportunidade de escrever este trabalho e deixarem marcas indeléveis em minha formação.

\section{Declaração}

Sem declarações ou interesse de conflitos

\section{Referências}

Baird DC, Meyers GJ, Hu JS. Testicular Cancer: Diagnosis and Treatment. American Family Physician, 97(4), 261-268, 2018.

Bosl GJ, Bajorin DF, Sheinfeld J. Cancer of the testis. In: DeVita VT Jr, Hellman S, Rosemberg SA, editors. Cancer: Principles and Practice of Oncology. 7 th ed. Philadelphia, PA: Lippincott Williams \& Wilkins, 1269-1293, 2005.

Cappuccio F, Rossetti S, Cavaliere C, Iovane G, Taibi R, D'Aniello C, Facchini G. Health-related quality of life and psychosocial implications in testicular cancer survivors: A literature review. European Review for Medical and Pharmacological Sciences, 22(3), 645-661, 2018.

Cook MB, Akre O, Forman D, et al. A systematic review and meta-analysis of perinatal variables in relation to the risk of testicular cancer-experiences of the son. International Journal of Epidemiology,39(6),1605-1618, 2010.

Dall'Oglio MF. Diagnóstico Diferencial das Massas Testiculares. Em: Nardozza Júnior A, Reis RB, Campos RSM, eds. Manual de Urologia. 1ed. São Paulo, Brasil: Editora Planmark, 95-101, 2010

Faria EF, Freitas Júnior CH. Câncer de Testículo. Em: Zerati Filho M, Nardozza Júnior A, Reis RB, eds. Urologia Fundamental. 1ed. São Paulo, Brasil: Editora Planmark, 179-188, 2010.

Horner MJ, Ries LAG, Krapcho M, et al. SEER cancer statistics review, 1975-2006. Bethesda (MD). Journal of the National Cancer Institute, 2009.

Maciel LC. Orquialgia Crônica. Em: Reis RB, Trindade Filho JCS, Simões FA, eds. Guia Rápido de Urologia. 1ed. São Paulo, Brasil: Editora Lemar, 166-169, 2012.

National Comprehensive Cancer Center. Testicular cancer: Version 2. Retrieved from January 10, 2018.
Presti JC. Tumores Genitais. Em: Mcaninch JW, Lue TF, eds. Urologia Geral de Smith e Tanagho. 18ed. Porto Alegre, Brasil: Editora Artmed, 379-391, 2014.

Silva LCZ. Câncer de Testículo. Em: Amaro JL, Tomé ALF, eds. Proteus: Palestras e Reuniões Organização para preparação ao Ttítulo de Especialista em Urologia SBU. 1ed. São Paulo, Brasil: Editora Planmark, 415420, 2017.

Smith AB, King M, Butow P, Luckett T, Grimison P, Tones $\mathrm{GC}$, Oliver I. The prevalence and correlates of supportive care needs in testicular cancer survivors: A cross-sectional study. Psycho-Oncology, 22(11), 2557-2564, 2013.

Srougi M. Câncer do Testículo. Em: Bendhack DA, Damião R, eds. Guia Prático de Urologia. 1ed. Rio de Janeiro, Brasil: Editora BG Cultural, 195-202, 1999.

Stephenson A, Gilligan TD. Neoplasias dos testículos. Em: Campbell SC, Lane BR, eds. Campbell-Walsh Urology. 11ed. Rio de Janeiro, Brasil: Editora Elsevier, 785-802, 2019.

Tavora JEF, Castro JCS, Lopes HE. Tumores Germinativos do Testículo: Epidemiologia, Classificação, Diagnóstico e Estadiamento. In: Nardi AC, Nardozza Júnior A, Bezerra CA, Fonseca CEC, Truzzi JC, Rios LAS, Sadi MV, eds. Urologia Brasil. 1ed. Rio de Janeiro, Brasil: Editora Planmark, 700-705, 2013.

Thompson JL, Blute ML. Coffee Ground Emesis: rare presentation of testicular cancer treated with neoadjuvant chemotherapy. Urology, 64(2), 376377, 2004.

Vlayen J, Vrijens F, Devriese S, Beirens K, Van Eycken E, Stordeur S. Quality indicators for testicular cancer: a population-based study. European Journal of Cancer, 48(8), 1133-1140, 2012.

Weijl NI, Rutten MF, Zwinderman AH, et al.

Thromboembolic events during chemotherapy for germ cell cancer: a cohort study and review of the literature. Journal of Clinical Oncology, 18, 21692178, 2000. 